\title{
O SEGUIMENTO DO GRADUADO EM ENFERMAGEM *
}

\author{
Cilene Aparecida Costardi Ide** \\ Katia Grillo Padilha** \\ Angela Maria Geraldo Pierin** \\ Sayuri Tanaka Maeda***
}

IDE, C.A.C.; PADILHA, K.G.; PIERIN, A.M.G.; MAEDA, S.T. O seguimento do graciuado em enfermagem. Rev. Esc. Enf. USP, São Paulo, 19(3):195-211, 1985.

Neste trabalho as autoras identificam as dificuldades encontradas pelos enfermeiros no inicio das atividades profissionais $e$ apresentam a avaliação desses profissionais quanto aos subsidios fornecidos pelos cursos de Graduação em Enfermagem.

\section{INTRODUÇÃO}

E compromisso comum, a docentes e profissionais do campo, avaliar até que ponto o curso de graduação em Enfermagem forma enfermeiros com bagagem teórico-prática suficiente e adequada para enfrentarem a realidade profissional.

DILASCIO (1970) salienta que o estudante, ao término do curso de enfermagem, não é um produto acabado, sendo que a formação recebida habilita-o apenas para o exercício inicial da sua carreira profissional. O estudante deverá estar consciente de tal limitação e o mercado de trabalho deverá assumir o compromisso em fornecer subsidios para o seu aperfeiçoamento. Essa mesma preocupação foi analisada no XXII Congresso Brasileiro de Enfermagem (1970), que teve como tema central a "Integração do Recém-Graduado em Enfermagem na vida profissional", onde foram levadas em conta as seguintes considerações:

- a integração do enfermeiro recém-graduado está diretamente relacionada à formação recebida, o que o tornará apto ou não para desempenhar papéis que dele são esperados;

* Trabalho apresentado no XXXVI Congresso Brasileiro de Enfermagem - Belo Horizonte, 1984. Publicação autorizada.

** Enfermeira. Mestre em Enfermagem. Professor Assistente do Departamento de Enfermagem Médico-Cirúrgica da Escola de Enfermagem da USP - disciplina Enfermagem Médico-Cirúrgica.

*** Enfermeira. Aluna do Curso de P6s-Graduação, nivel Mestrado, da Escola de Enfermagem da USP. 
modelo;

- as docentes e enfermeiras do campo desempenham papéis de

- a insegurança do jovem enfermeiro poderá ser amenizada, se as escolas demonstrarem interesse no trabalho que ele desenvolverá no campo, para auxiliá-lo nas dificuldades encontradas.

A partir desse momento, evidencia-se uma preocupação nas Escolas de Enfermagem em realizar mudanças que favoreçam a adequação da formação do profissional baseada na realidade sócio-econômico - político-cultural - educacional de saúde e da profissão. Para tanto, foram organizados grupos de estudo visando mudanças curriculares nos cursos de graduação em Enfermagem, o que se fez sentir por exemplo na Escola Ana Neri, que iniciou seus estudos em 1976 e, atualmente, na Escola de Enfermagem da USP.

Essa abordagem é fundamentada também nas considerações citadas por KRAUZ (1977), que aponta, dentre as múltiplas funções da Universidade, a de atender as necessidades presentes e futuras da sociedade na qual está inserida, inclusive a de buscar novas soluções para os problemas existentes, por meio de pesquisas e formação de recursos humanos capazes de utilizarem conhecimentos e práticas que atendam as necessidades de mudanças sócio-econômicas.

CAMPOS apud KAMIYAMA (1981) afirma que "o ensino profissional deve ser o precursor de novas tendências e aspirações científicoculturais e o professor o líder do processo educacional".

TRALDI (1977), ao considerar o planejamento curricular, salienta a necessidade de serem efetuados o levantamento prévio da situação, o planejamento e a organização dos recursos necessários, bem como a avaliação no decorrer e no final do processo; há necessidade, ainda, de verificação das consequiências ou reflexos nos indivíduos que passaram pelo processo e da sua repercussão na sociedade. Essa afirmação enfatiza a relevância do seguimento do graduado, como base para se saber se os egressos da instituição estão se adaptando, fora da escola, a seu trabalho e a vida profissional.

Verifica-se, no dia a dia, o testemunho de vários enfermeiros em relatar o impacto que sofrem no início da carreira profissional, na qual encontram dificuldades no exercício de atividades especificas de enfermagem, incongruências entre seus anseios e as propostas feitas pelo mercado de trabalho.

ALHADEFF (1979), ao falar sobre a ansiedade do recém-graduado em enfermagem, ressalta que este encontrará um mundo com realidades que muitas vezes trarão dificuldades para o início do exercício profissional; destaca que a habilidade se adquire ao repetir uma tarefa várias vezes e que a confiança e a responsabilidade se desenvolvem a partir do momento em que se domina o medo e se enfrentam as situações novas; este medo decorre da falta de conhecimento, sendo a escola a culpada em não apontar o caminho a ser seguido após o término do curso. 
Considerando que esses fatores podem estar relacionados à bagagem de conhecimentos teórico-práticos do enfermeiro, adquirida durante a sua formação, e através do exercício profissional, o presente estudo tem como objetivos:

1 - detectar quais as dificuldades encontradas pelo(a) enfermeiro(a) no início da sua atividade profissional;

2 - identificar quais as expectativas do(a) enfermeiro(a) quanto ao início da sua atividade profissional;

3 - identificar o grau de satisfação do(a) enfermeiro(a) em relação ao seu desempenho profissional;

4 - averiguar a avaliação que o(a) enfermeiro(a) faz dos subsídios oferecidos pelos cursos de graduação para o início da sua atividade profissional.

\section{MATERIAL E MÉTODO}

O presente estudo foi realizado em um hospital geral, particular, do Município de São Paulo.

\section{População}

A população deste trabalho é composta por 31 enfermeiras, o que representa $48,4 \%$ do total de enfermeiras existentes no hospital utilizado como campo para esta investigação.

\section{Critérios de Seleção} lação:

Os seguintes critérios foram estabelecidos para a seleção da popu-

- enfermeiras formadas de zero a seis anos antes do início da pesquisa;

- enfermeiras incluídas na escala normal de trabalho do hospital por ocasião da coleta de dados, ou seja, que não estivessem gozando férias, licença ou afastamento por qualquer natureza.

\section{Variáveis do estudo}

\section{- Variáveis independentes}

- tempo decorrido após a formatura.

- cursos realizados.

- Variáveis dependentes

- dificuldades para conseguirem emprego como enfermeira;

- dificuldades para desempenharem as atividades de enfermagem no início da carreira;

- atividades de enfermagem que esperavam executar no início da da carreira;

- atividades de enfermagem que executam atualmente;

- nivel de satisfação quanto ao desempenho profissional;

- avaliação dos subsídios fornecidos pelo curso de graduação. 
Coleta de dados

A coleta de dados foi realizada na $1^{\text {a }}$ quinzena de maio de 1983.

Para essa coleta foram utilizados os seguintes instrumentos: carta explicativa às enfermeiras, solicitando colaboração na pesquisa (Anexo I), e questionário específico a ser respondido com perguntas abertas e fechadas, constituído de 2 partes: Parte I - caracterização da população quanto ao sexo, idade, tempo decorrido e cursos realizados após a formatura; Parte II - relacionada aos objetivos do trabalho: dificuldades para conseguir emprego, atividades que esperava encontrar ao início do exercício profissional e as que executa atualmente, dificuldades encontradas no desempenho das atividades profissionais e avaliação dos subsídios fornecidos pelo curso de graduação.

Operacionatização da coleta de dados

A coleta de dados foi realizada após a autorização da Chefia do Serviço de Enfermagem do hospital, sendo feita pelas pesuisadoras que entraram em contacto com a enfermeira responsável pela Educação em Serviço, a qual se incumbiu da distribuição e do recolhimento dos questionários. Todos os questionários distribuídos foram respondidos e devolvidos dentro do prazo estabelecido de dez dias contados a partir da data da entrega.

\section{RESULTADOS}

A análise dos resultados foi feita em números absolutos e percentuais.

TABELA 1

Distribuição da população segundo idade e tempo decorrido após a formatura São Paulo, 1983

\begin{tabular}{|c|c|c|c|c|c|c|c|c|}
\hline \multirow{3}{*}{ Idade } & \multicolumn{6}{|c|}{ Tempo em anos apos a formatura } & \multirow{2}{*}{\multicolumn{2}{|c|}{ Total }} \\
\hline & \multicolumn{2}{|c|}{$0-12$} & \multicolumn{2}{|c|}{$2-14$} & \multicolumn{2}{|c|}{$4-16$} & & \\
\hline & $\mathbf{n}^{\circ *}$ & $\% * *$ & $\mathbf{n}^{9}$ & $\%$ & $\mathbf{n}^{9}$ & $\%$ & & \\
\hline $201-30$ & 14 & 45,2 & 9 & 29,0 & 5 & 16,1 & 28 & 90,3 \\
\hline $30 \longmapsto 40$ & - & - & - & - & 2 & 6,4 & 2 & 6,4 \\
\hline $401-+$ & - & - & - & - & 1 & 3,3 & 1 & 3,3 \\
\hline Total & 14 & 45,2 & 9 & 29,0 & 8 & 25,8 & 31 & 100,0 \\
\hline
\end{tabular}

$* \mathrm{n}^{\circ}=$ frequêencia absoluta

** $\%=$ frequiência relativa

Os dados da Tabela 1 revelam que 90,3\% das enfermeiras situam-se na faixa etária entre 20 e 30 anos. 
Do total da amostra, $45,2 \%$ das enfermeiras haviam se formado entre 0 e 2 anos antes deste estudo, $29,0 \%$ entre 2 e 4 anos e $25,8 \%$ entre 4 e 6 anos.

\section{TABEIAA 2}

Distribuição da população segundo a existência ou não de dificuldade em conseguir emprego. São Paulo, 1983

\begin{tabular}{c|cc}
\hline $\begin{array}{c}\text { Dificuldade em } \\
\text { conseguir emprego }\end{array}$ & no & $\%$ \\
\hline sim & 14 & 45,2 \\
não & 17 & 54,8 \\
\hline TOTAL & 31 & 100,0 \\
\hline
\end{tabular}

Pela Tabela $2,54,8 \%$ das enfermeiras não encontraram dificuldade em conseguir emprego. Observa-se, entretanto, que número significativo cie enfermeiros $(45,2 \%)$ referiram dificuldades que são relacionadas na tabela seguinte.

\section{TABELA 3}

Relação das dificuldades referidas pelas enfermeiras em conseguirem emprego. São Paulo, 1983

\begin{tabular}{l|cc}
\hline \multicolumn{1}{c|}{ Dificuldades em conseguirem emprego } & n' & $\%$ \\
Número de vagas & 7 & 26,9 \\
Exigência de experiência anterior & 6 & 23,1 \\
Nivel salarial oferecido & 6 & 15,4 \\
Exigência do curso de habilitação & 4 & 11,5 \\
Outros & 3 & 100,0 \\
\hline TOTAL & 26 & \\
\hline
\end{tabular}

A média de dificuldades em conseguir emprego foi de $1,85 \%$ por enfermeira (Tabelas 2 e 3 ).

Pela Tabela 3 constata-se que a maior dificuldade referida $(26,9 \%)$ diz respeito ao número de vagas existentes, seguido da exigência de experiência anterior $(23,1 \%)$ e nível salarial oferecido $(23,1 \%)$. Esclarece se, ainda, que dentro de "Outros" foram incluídas as seguintes dificuldades: ser casada, demora para convocação e referências dadas pela escola. 
Relação das expectativas referidas pelas enfermeiras quanto às atividades que esperava executar. São Paulo, 1983

\begin{tabular}{l|rr}
\hline \multicolumn{1}{c|}{ Atividades que esperavam executar } & ne & $\%$ \\
\hline Assistenciais diretas & 31 & 62,0 \\
Burocrático-administrativas & 14 & 28,0 \\
Ensino & 5 & 10,0 \\
\hline \multicolumn{1}{c}{ TOTAL } & 50 & 100,0 \\
\hline
\end{tabular}

Observa-se pela Tabela 4 que a atividade assistencial corresponde a $62 \%$ das expectativas referidas.

\section{TABELA 5}

Relação das atividades executadas pelas enfermeiras. São Paulo, 1983

\begin{tabular}{l|rr}
\hline \multicolumn{1}{c|}{ Atividades executadas } & $\mathrm{n} \%$ & $\%$ \\
\hline Assistenciais diretas & 25 & 59,5 \\
Burocrático-administrativas & 16 & 38,0 \\
Ensino & 1 & 2,5 \\
\hline \multicolumn{1}{c|}{ TOTAL } & 42 & 100,0 \\
\hline
\end{tabular}

Os dados da Tabela 5 mostram que a atividade assistencial direta é a mais apontada $(59,5 \%)$, seguida da atividade administrativa $(38,0 \%)$. Cabe ressaltar que existe compatibilidade entre as expectativas citadas e as atividades desempenhadas pelas enfermeiras na prática profissional.

TABELA 6

Distribuição da população segundo existência ou não de dificuldades encontradas no início do exercício profissional. São Paulo, 1983

\begin{tabular}{c|cc}
\hline $\begin{array}{l}\text { Dificuldades encontradas no } \\
\text { inficio do exencício }\end{array}$ & $\mathrm{n}^{\circ}$ & $\%$ \\
\hline Sim & 20 & 64,5 \\
Não & 11 & 35,5 \\
\hline TOTAL & 31 & 100,0 \\
\hline
\end{tabular}

Pela Tabela 6 verifica-se que a maioria das enfermeiras $(64,5 \%)$ referiu dificuldades para o desempenho das atividades profissionais, no inicio da sua carreira. 
Relação das dificuldades encontradas pelas enfermeiras no início do exercício profissional. São Paulo, 1983

Dificuldades encontradas no início do exercicio profissional n $\%$

Falta de correlação entre a teoria e a prática

Falta de aceitação das funções institucionais pré-estabelecida

Falta de habilidade técnica

Dificuldade no relacionamento interprofissional

Dificuldade no relacionamento com a equipe de enfermagem

Dificuldade no relacionamento com pacientes

\begin{tabular}{l|rl}
\hline & $\mathrm{n}^{\mathrm{q}}$ & $\%$ \\
\hline & $\left.\begin{array}{rl}14 & 29,8 \\
9 & 19,2 \\
8 & 17,0 \\
8 & 17,0 \\
6 & 12,7\end{array}\right] 29,7$ \\
2 & 4,3 \\
\hline
\end{tabular}

Das 20 profissionais que apontaram dificuldades no inicio do exercício profissional, constatou-se uma média de 2,3 dificuldades por enfermeira (Tabela 6 e 7).

Os dados da Tabela 7 demonstram que a maior dificuldade indicada pelas enfermeiras no que se refere a sua formação profissional é a correlação entre teoria e prática $(29,8 \%)$ seguida da falta de habilidade técnica $(17,0 \%)$. Evidencia-se ainda, que a dificuldade de aceitação das funções pré-estabelecidas $(19,2 \%)$ assim como as encontradas no relacionamento profissional $(29,7 \%)$ são relativas a aspectos institucionais referidos em maior número pelas enfermeiras.

TABELA 8

Distribuição da população segundo nível de satisfação referente ao desempenho profissional. São Paulo, 1983

\begin{tabular}{l|cc}
\hline Nivel de satisfacão & $n^{\circ}$ & $\%$ \\
\hline Satisfeito & 25 & 80,6 \\
Pouco satigfeito & 06 & 19,4 \\
\hline \multicolumn{1}{c|}{ TOTAL } & 31 & 100,0 \\
\hline
\end{tabular}

Observa-se pela Tabela 8 que a grande maioria das enfermeiras $(80,6 \%)$ está satisfeita com o seu desempenho profissional.

Cabe ressaltar que nenhuma enfermeira referiu estar insatisfeita quanto ao desempenho profissional. 
Distribuição da população segundo a avaliação das subsídios oferecidos pelo curso de graduação. São Paulo, 1983

\begin{tabular}{l|cc}
\hline \multicolumn{1}{c|}{ Subsidios } & $\mathbf{n}^{\circ}$ & $\%$ \\
\hline Suficientes & 14 & $\mathbf{4 5 , 2}$ \\
Suficientes em parte & 13 & $\mathbf{4 2 , 0}$ \\
Insuficientes & 03 & $\mathbf{9 , 6}$ \\
Em branco & 01 & $\mathbf{3 , 2}$ \\
\hline \multicolumn{1}{c|}{ TOTAL } & 31 & 100,0 \\
\hline
\end{tabular}

Pela Tabela 9 verifica-se que $45,2 \%$ dos enfermeiros consideraram os subsídios oferecidos pela escola como suficientes, embora seja pequena a diferença entre aqueles que os consideraram como suficientes apenas em parte $(42,0 \%)$. Cabe ressaltar que apenas três enfermeiras (menos de $10 \%$ ) consideraram insuficiente o ensino ministrado pela escola.

TABEYLA 10

Distribuição da população segundo o tempo decorrido após a formatura e dificuldade ou não em conseguir emprego. São Paulo, 1983

\begin{tabular}{|c|c|c|c|c|c|c|}
\hline \multirow{3}{*}{$\begin{array}{l}\text { Tempo após } \\
\text { a formatura }\end{array}$} & \multicolumn{4}{|c|}{ Dificuldade em conseguir emprego } & \multirow{2}{*}{\multicolumn{2}{|c|}{ Total }} \\
\hline & \multicolumn{2}{|c|}{$\operatorname{sim}$} & \multicolumn{2}{|c|}{ não } & & \\
\hline & $\mathrm{n}^{9}$ & $\%$ & $\mathrm{n}^{9}$ & $\%$ & $\mathrm{n}^{\circ}$ & $\%$ \\
\hline $0-12$ & 6 & 46,2 & 7 & 53,8 & 13 & 100,0 \\
\hline $0 \longrightarrow 4$ & 6 & 60,0 & 4 & 40,0 & 10 & 100,0 \\
\hline $0 \ldots-\mid 6$ & 2 & 25,0 & 6 & 75,0 & 8 & 100,0 \\
\hline TOTAL & 14 & 45,2 & 17 & 54,8 & 31 & 100,0 \\
\hline
\end{tabular}

Os dados da Tabela 10 revelam que $75 \%$ das enfermeiras com tempo após a formatura de $4-6$ anos não relatam dificuldades para conseguir emprego.

Observa-se, também, que, na faixa de $2-4$ anos, há quase inversão daqueles dados, enquanto que na de 0 - 2 anos a maior parte das enfermeiras $(53,8 \%)$ relatou não ter tido dificuldade em conseguir emprego. 
Distribuição da população segundo a compatibilização entre as atividades que esperava executar c as que executa atualmente. São Paulo, 1983

\begin{tabular}{l|rc}
\hline Compatibilização & n & $\%$ \\
\hline Parcial & 20 & 64,5 \\
Total & 9 & 29,0 \\
Nenhuma & 2 & 6,5 \\
\hline TOTAL & 31 & 100,0 \\
\hline
\end{tabular}

A compatibilização total e a parcial foi resultante de uma análise comparativa entre as atividades que esperava executar e as que executa atualmente. Foi considerada total quando todos os itens apontados foram coincidentes e parcial quando apenas alguns itens se repetiam nas duas questões.

Assim, pela Tabela 11 verifica-se que apenas $29 \%$ das enfermeiras realizam as atividades que esperavam executar no inicio da carreira profissional, enquanto que a maior parte $(64,5 \%)$ executa apenas parcialmente as atividades de acordo com sua expectativa. Cabe ressaltar que para $6,5 \%$ das enfermeiras não houve compatibilização alguma.

TABELA 12

Distribuição da população segundo a compatibilização entre as atividades que esperava executar e as que executa atualmente e o nível de de satisfação referente ao desempenho profissional. São Paulo, 1983

\begin{tabular}{|c|c|c|c|c|c|c|}
\hline \multirow{3}{*}{ Compatibilizacão } & \multicolumn{4}{|c|}{ Nivel de satisfação } & \multirow{2}{*}{\multicolumn{2}{|c|}{ Total }} \\
\hline & \multicolumn{2}{|c|}{ satisfeito } & \multicolumn{2}{|c|}{ pouco satisfeito } & & \\
\hline & $n^{*}$ & $\%$ & $\mathbf{n}^{\circ}$ & $\%$ & $\mathrm{n}^{\mathbf{0}}$ & $\%$ \\
\hline Parcial & 15 & 75,0 & 5 & 25,0 & 20 & 100,0 \\
\hline Total & 9 & 100,0 & - & - & 9 & 100,0 \\
\hline Nenhuma & 01 & 50,0 & 1 & 50,0 & 2 & 100,0 \\
\hline TƠTAL & 25 & 80,6 & 6 & 19,4 & 31 & $.100,0$ \\
\hline
\end{tabular}

Os dados da Tabela 12 demonstram que dos enfermeiros que referiram compatibilização parcial entre as atividades que esperavam executar e as que executam, $15(75,0 \%)$, encontram-se satisfeitos e $5(25,0 \%)$ estão pouco satisfeitos. E importante ressaltar que os 9 enfermeiros que referiram compatibilização total estão satisfeitos. 
Distribuição da população segundo os subsidios fornecidos pelo curso de graduação e as dificuldades encontradas no início do exercício profissional. São Paulo, 1983

\begin{tabular}{|c|c|c|c|c|c|c|}
\hline \multirow{3}{*}{ Subsidios } & \multicolumn{4}{|c|}{ Dificuldades no inicio do exercício } & \multirow{2}{*}{\multicolumn{2}{|c|}{ Total }} \\
\hline & \multicolumn{2}{|c|}{$\operatorname{sim}$} & \multicolumn{2}{|c|}{ não } & & \\
\hline & ne & $\%$ & $\mathbf{n}^{\circ}$ & $\%$ & ne & $\%$ \\
\hline \multirow{2}{*}{$\begin{array}{l}\text { Suficientes em parte } \\
\text { Suficientes }\end{array}$} & 11 & 78,6 & 3 & 21,4 & 14 & 100,0 \\
\hline & 6 & 46,2 & 7 & 53,8 & 13 & 100,0 \\
\hline Insuficientes & 3 & 100,0 & - & - & 3 & $10 c, 0$ \\
\hline Em branco & 一 & 一 & 1 & 100,0 & 1 & 100,0 \\
\hline TOTAL & 20 & 64,5 & 11 & 35,5 & 31 & 100,0 \\
\hline
\end{tabular}

Pela Tabela 13 verifica-se que, dos 14 enfermeiros que consideraram os subsídios fornecidos pelo curso de graduação como suficientes em parte $11(78,6 \%)$ relataram dificuldades no exercício profissional. Nota-se também que dos 13 enfermeiros que consideraram os subsídios suficientes, a maioria, $(53,8 \%$ ) não encontrou dificuldades. Ressalta-se, ainda, que os 3 enfermeiros que consideraram insuficientes os subsídios encontraram dificuldades no início das atividades profissionais.

TABELA 14

Distribuição da população segundo o tempo após a formatura e o nivel de satisfação referente ao desempenho profissional. São Paulo, 1983

\begin{tabular}{|c|c|c|c|c|c|c|}
\hline \multirow{3}{*}{ Tempo de formada } & \multicolumn{4}{|c|}{ Nivel do satisfacão } & \multirow{2}{*}{\multicolumn{2}{|c|}{ Total }} \\
\hline & \multicolumn{2}{|c|}{ satisfeito } & \multicolumn{2}{|c|}{ pouco satisfeito } & & \\
\hline & $\mathbf{n}^{\circ}$ & $\%$ & $\mathrm{n}^{\varphi}$ & $\%$ & $n^{\varphi}$ & $\%$ \\
\hline $0-12$ & 11 & 78,6 & 3 & 21,4 & 14 & 100,0 \\
\hline $2--14$ & 7 & 77,7 & 2 & 22,3 & 9 & 100,0 \\
\hline $4-16$ & 7 & 87,5 & 1 & 12,5 & 8 & 100,0 \\
\hline TOTAL & 25 & 80,6 & 6 & 19,4 & 31 & 100,0 \\
\hline
\end{tabular}

Os dados da Tabela 14 evidenciam que a maioria da população $(80,6 \%)$ encontra-se satisfeita quanto ao desempenho profissional, independente do tempo decorrido após a formatura. 
Distribuição da população segundo os cursos realizados e as dificuldades encontradas ou não no início do exercício profissional. São Paulo, 1983

\begin{tabular}{|c|c|c|c|c|c|c|}
\hline \multirow{3}{*}{ Cursos realizados } & \multicolumn{4}{|c|}{ Dificuldades no início } & \multirow{2}{*}{\multicolumn{2}{|c|}{ Total }} \\
\hline & \multicolumn{2}{|c|}{ sim } & \multicolumn{2}{|c|}{ não } & & \\
\hline & n' & $\%$ & $\mathbf{n}^{9}$ & $\%$ & $\mathbf{n}^{9}$ & $\%$ \\
\hline Graduaço & 4 & 66,6 & 2 & $\mathbf{3 3 , 4}$ & 6 & 100,0 \\
\hline Graduação e outros & 16 & 64,0 & 9 & 36,0 & 25 & 100,0 \\
\hline TOTAL & 20 & 64,5 & 11 & 35,5 & 31. & 100,0 \\
\hline
\end{tabular}

Pela Tabela 15 verifica-se que a maioria da população $(64,5 \%$; encontrou dificuldades no início do exercicio profissional, independentemente dos cursos realizados.

\section{DISCUSSÃO}

A população no presente estudo constituiu-se quase exclusivamente por enfermeiras na faixa etária entre 20 e 30 anos, a maioria até dois anos antes do estudo, caracterizando-se, ainda, por terem feito outros cursos além da graduação (Tabelas 1 e 15).

No que diz respeito à dificuldade em conseguir emprego, verifica-se pela Tabela 2, que a maioria não relata dificuldades, embora seja bastante significativo o número de enfermeiras que as referem. Essa dificuldade, quando correlacionada ao tempo após a formatura se faz sentir pelas enfermeiras graduadas entre 2 e 4 anos antes do estudo e pelas recém-formadas (Tabela 10).

Quanto à especificação das dificuldades, evidencia-se que aquelas referentes aos aspectos institucionais se destacam, conforme os resultados da Tabela 3. Assim, a análise desses dados sugere que as dificuldades estão mais relacionadas ao mercado de trabalho que ao órgão formador, o que parece refletir a atual situação econômica do nosso meio que limita a oferta de empregos.

No tocante às expectativas no início do exercício profissional, nota-se que a maioria das enfermeiras espera executar atividades assistenciais diretas o que coincide com a sua prática profissional (Tabelas 4 e 5). Verifica-se, ainda, pela Tabela 11, que a compatibilização entre aquelas atividades é apenas parcial para a maioria das enfermeidas.

Esses resultados parecem demonstrar que a formação profissional, predominantemente relacionada à assistência curativa, forma o individuo com expectativas voltadas para a assistência direta, o que coincide com a sua prática hospitalar. 
A análise dessa situação reflete uma aparente coerência entre a formação universitária e o mercado de trabalho, contudo, se analisar as exigências atuais da política nacional de saúde, voltada para a assistência primária basicamente, depara-se com o seguinte questionamento: a quem deve a escola satisfazer?

Essa preocupação aparece de forma evidente nos dizeres de KAMIYAMA (1981), que aborda a necessidade de modificação dos currículos universitários de enfermagem em face das novas tendências de saúde, diretamente voltadas para a extensão de cobertura dos serviços básicos.

Apesar da coerência apontada anteriormente, as enfermeiras ainda referem dificuldades na prestação da assistência ao paciente, no início do exercício profissional, as quais estão relacionadas tanto à correlação teórico-prática e à falta de habilidade técnica, que deveriam ter sido adquiridas na escola, como à não aceitação das funções institucionais pré-determinadas e ao relacionamento inter-profissional, inerentes à instituição (Tabelas 6 e 7).

Quanto ao nível de satisfação referente ao desempenho profissional, evidencia-se que a maioria das enfermeiras está satisfeita independentemente do tempo decorrido após a formatura (Tabelas 8 e 14), talvez em virtude das condições de trabalho oferecidas pela instituição. Quanto àquelas que referem sentir-se pouco satisfeitas, as justificativas recaem na incompatibilidade das expectativas e na não aceitação das atribuições pré-determinadas pela instituiçã.o

Tais fatos vêm ao encontro das colocações feitas por CHOI-LAO \& LOGAN (1979) que apontam a existência de valores diferentes entre o "mundo de trabalho" e o "mundo da escola" como fontes geradoras de grande preocupação para a enfermeira recém-graduada.

Do mesmo modo, KRAMER apud FINKELSTEIN (1979), analisa a crise de identidade do recém-graduado ao se deparar com a incongruência entre o sistema de valores da instituição e aqueles aprendidos na escola.

$E$ interessante salientar que, apesar da maioria das enfermeiras referir compatibilização apenas parcial entre as atividades que esperava executar e as que executa atualmente, um maior número delas aponta satisfação quanto ao desempenho profissional (Tabela 12).

Essa incoerência poderia ser explicada por suposições como: despreparo profissional, para servir como agente modificador e estabilizador de situações, levando a uma aceitação de papéis pré-estabelecidos pela instituição; situação sócio-econômica atual, levando a instabilidade de emprego que, conseqüentemente, levaria à acomodação, com poucas reivindicações, ou, ainda, como aspecto positivo, um campo de trabalho suficientemente rico, que supre as deficiências decorrentes da formação universitária.

No que se refere à avaliação dos subsídios fornecidos pela escola, os dados da Tabela 9 demonstram proximidade entre o número de enfermeiras que os considerou suficientes e aquelas que os apontaram como suficientes apenas em parte. 
Vale a pena ressaltar que, de acordo com as respostas obtidas, independentemente dos subsídios serem considerados suficientes ou suficientes apenas em parte, observa-se certa uniformidade quanto às justificativas apresentadas: a falta de correlação entre teoria e prática, campo de estágio pobre em experiências e o despreparo de docentes como fatores limitantes ao desenvolvimento de estudante.

Referente ao último ítem citado, KAMIYAMA (1981) ressalta que "aceitar com espírito crítico e avaliar os desafios e problemas que se sucedem é tarefa fundamental do professor, que, não deve olvidar a grande responsabilidade que the cabe na direção do ensino e do progresso da profissão, visto que do grupo docente depende em grande parte o destino dessa profissão". Dessa maneira, tem-se enfatizada a necessidade relevante do preparo do docente para que possa participar efetivamente do processo ensino-aprendizagem.

Nota-se que os dados obtidos na Tabela 9 são coerentes com os da Tabela 13, os quais demonstram que a maioria da população, incluindo os que referiam os subsidios como suficientes, encontrou dificuldades no início do exercício profissional. Verifica-se, ainda, que as dificuldades existem no início do exercício profissional independentemente, inclusive, dos cursos realizados (Tabela 15).

Cabe aqui questionar se o órgão formador seria o único responsável pelo acabamento final do profissional, uma vez que, segundo KRAUSZ (1977), por mais que a Universidade se ajuste às necessidades sociais presentes, dificilmente poderá chegar a formar um profissional sob medida para um setor do mercado de trabalho. De acordo com esse autor, essa responsabilidade estende-se, também, às instituições empregadoras, através de treinamento em serviço adequado às funçōes que o indivíduo irá assumir.

\section{CONCLUSOES}

Os resultados do estudo realizado com $48,4 \%$ dos enfermeiros da instituição hospitalar utilizada por esta investigação conduziram às conclusões abaixo.

- As dificuldades para conseguir emprego no inicio da atividade profissional, referidas por $45,2 \%$ das enfermeiras, estão relacionadas predominantemente às exigências institucionais.

- As dificuldades encontradas no início do exercício profissional referidas por $64,5 \%$ das enfermeiras são:

- falta de correlação entre a teoria e prática;

- falta de habilidade para a execução das tarefas;

- falta de habilidade no desempenho de funções pré-estabelecidas pela instituição hospitalar.

As atividades assistenciais diretas foram as mais apontadas $(62,0 \%)$ como aquelas a serem desempenhadas no inicio do exercício profissional 
e são também as mais executadas $(59,5 \%)$. Apesar disso, pera a maioria $(64,5 \%)$ existe compatibilização apenas parcial entre aquelas atividades.

A maioria das enfermeiras $(80,6 \%)$ está satisfeita com o seu desempenho profissional, independentemente do tempo decorrido após a formatura e apesar da compatibilização apenas parcial entre as atividades que esperava executar e as que realmente executa.

Os subsídios oferecidos pelo curso de graduação para o início da atividade profissional foram considerados como suficientes por 45,2\% das enfermeiras e como suficientes em parte por $42,0 \%$ da população. Apesar disso, a maioria $(64,5 \%)$ referiu dificuldades no início do exercício profissional, que parecem independer dos cursos realizados.

IDE, C.A.C.; PADILHA, K.G.; PIERIN, A.M.G.; MAEDA, S.T. The following of the graduated in nursing. Rev. Esc. Enf. USP, São Paulo, 19(3):195-211, 1985.

In this study the authors identify the nurses' difficulties at the begining of their professional activities, and evaluate the type of education offered by their undergraduate nursing program.

\section{REFERENCIAS BIBLIOGRAFICAS}

ALHADEFF, G. Anxiety in a new graduate. Am. J. Nurs., New York, 79(4):688-8, Apr. 1979.

CHOI-LAO, A.T.U. \& LOGAN, M.S. Bridging the gap between education and service. Can. Nurse, Montreal 75(3):34-5, Mar. 1979.

DILASCio, C.M.D. Integração do recém-nascido na vida profissional. Rev. Bras. Enf., Rio de Janeiro, 23(2):57-72, Jan./Jun. 1970.

FINKELSTEIN, R. Reflections of a former new graduate. Imprint, New York, 26(5):50-1, Dec. 1979.

KAMIYAMA, Y. Considerações sobre o perfil do graduado em enfermagem. Rev. Esc. Ens. USP, São Paulo, 15(2):211-21, ago. 1981.

KRAUSZ, R.R. Algumas reflexס̃es sobre a formação de recursos humanos para a saúde pública. Rev. Saúde Pábl., São Paulo, 11:151-55, 1977.

TRALDI, L.L. O follow up e sua importancia no planejamento curricular. Educação, Brasilla, 6(23) :23-7, Jan./mar. 1977 . 


\section{ANEXO I}

São Paulo, de de 1983

Prezado(a) colega

Nós, alunas de Pós-Graduação do Curso de Mestrado da Escola de Enfermagem da USP, estamos interessados em identificar quais as dificuldades enfrentadas por voo no inicio de seu exercício profissional, assim como a avaliação que faz dos subsídios oferecidos pcla Escola para enfrentá-las.

Acreditando que os dados fornecidos por vocé serão de grande valia para possiveis modificações currículares, solicitamos a sua colaboração no preenchimento deste questionário.

Desde já, agradecemos sua atenção. 


\section{ANEXO II}

1. Sexo

$\begin{array}{lll}\text { Masculino } & (\quad) \\ \text { feminino } & (\quad)\end{array}$

2. Idade

anos completos

3. Formatura

Ano

4. Cursas realizados

\begin{tabular}{|c|c|c|}
\hline graduação & ( & \\
\hline habilitação & ( & \\
\hline especialização & ( & \\
\hline
\end{tabular}

5. Vocé encontrou dificuldade para conseguir emprego como enfermeiro(a), no inicio de sua atividade profissional?

não encontrel ( )

encontrei e foram relacionadas a:

- número de vagas ( )

- exigência de experiência anterior como enfermeiro(a) ( )

- exigência do curso de habilitação ( )

- nivel salarial oferecido ( )

- outras ( ) Quais?

6. No início de seu exercício profissional, que atividades esperava executar? atividades docentes nos variados níveis ( )

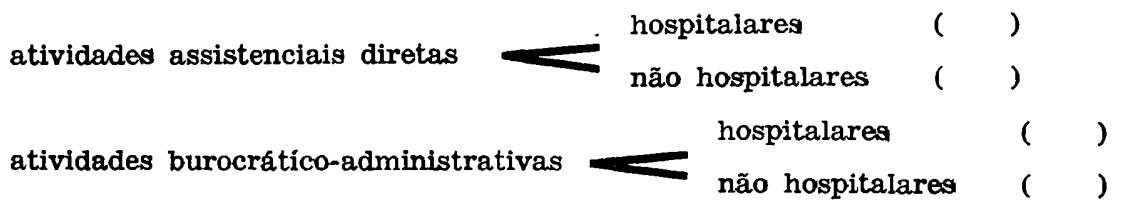
outras ( ) Quais? 
7. Que atividades você realmente executa?

(Identifique quantas alternativas forem necessárias)

atividadies docentes em vários níveis ( )

atividades assistenciais diretas ( )

atividades burocrático-administrativas ( )

Especifique:

outras ( ) Quais:

8. No inicio do seu exercício profissional, você encontrou dificuldades para desempenhar as atividades que lhe eram pertinentes?

não encontrei ( )

encontrei em relação a:

(assinale todas as encontradas)

- habilidades na execução de técnicas de enfermagem ( )

- correlação da teoria à prática ( )

- aceitação das funções pré-determinadas pela instituição ( )

- relacionamento interprofissional ( )

- relacionamento com elementos da equipe de enfermagem ( )

- relacionamento com pacientes e/ou cliente e familia ( )

- outras ( ) Quais:

9. Como você se sente com relação ao seu desempenho profissional?

satisfeito

pouco satisfeito

insatisfeito

Justifique a alternativa assinalada:

10. Os subsidios fornecidos pelo seu curso de graduação para a execução das atividades desempenhadas foram:

suficientes

suficientes em parte

insuficientes

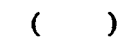

$(\quad)$

()

